\title{
Archetypes of art and concepts of science as pathways from natural to engineering design
}

\author{
A. A. Berezin \\ Department of Engineering Physics, McMaster University, Canada
}

\begin{abstract}
In a broad sense "engineering" means any activity aimed at the creation of material-based artifacts to meet various human needs and interests. In all areas of engineering, interdisciplinary and multidisciplinary connections play a significant role. Aesthetical aspects and analogies taken from arts and concepts of science (including ideas of quantum physics) often serve as stimuli for engineering design. Of a particular interest are imports from surrealistic and paradoxical ("visionary") arts, as well as some radical ideas and concepts of physics. This article discusses, often overlooked and subtle, inputs to engineering design from the realms of artistic creativity and concepts relevant to microphysical (quantum) levels of physical reality.
\end{abstract}

Keywords: engineering design, esthetics, visionary art, virtual reality, surrealism, complexity and chaos theory, quantum physics, set theory, information, spontaneous pattern formation.

\section{Introduction}

Under the most encompassing view, the term "engineering" refers to the creation of any material-based artifacts that target, in one way or the other, some objectives of human existence. Encompassing some 14 orders of magnitude from nanotechnology to major terrestrial constructions and ocean ships products of engineering are often influenced in their gestation and development by the esthetical, cultural and philosophical views of their creators.

This paper discusses some plausible connections between engineering design (ED) and technology on one hand, and the realms of art and science on the other. The very origin of the terms "engineering" (creative design) and "technology" are rooted in the notion of artistic activity (Greek techne: art, craft, skill). As was 
mentioned by Aristotle, the question "why to make" (final causation) is always superior to the "how to do" question - this generally remains so for both ED and arts.

At the same time, both science and arts have elements of transcendence and pure curiosity aimed at the attempts to comprehend "how it (the world) works". Often science and art are dealing with notions of infinity and the infinite resourcefulness of the cosmos, as discussed in more detail further in this paper. All this, in various degrees and fashions, can affect engineering mentality and hence be reflected in the products of ED.

It should be said, though, that practitioners of ED rarely, if ever, make any explicit statements that they were influenced by this-or-that type of art, or a specific piece of art, in their inventive and design work. Examples mentioned in this paper are personal selections of the author and hence are subjective. Likewise, the proposed conclusions and inferences are merely suggestive. The author believes that it is justified by the prime aim of this article, which is to stimulate further thinking about the connection between arts, science and technology.

\section{Arts and technology}

It is often stated that the relationship between the worlds of arts and technology is peculiar and complicated. On one hand, history gives impressive examples when top artistic creativity productively co-existed in single individuals. It is enough to recall such names as Leonardo da Vinci (1452-1519) or Albrecht Durer (1471-1528). However, as we move from the Renaissance times - times of integrated knowledge - to our, utterly diversified, modern age - things become more mixed and tenuous.

\subsection{Hard and soft disciplines}

Anyone familiar with modern university campus and university life will not fail to notice that the interaction between "hard" disciplines (physics, chemistry, mathematics, engineering) and "soft" areas (humanities, sociology, political sciences, visual and performing arts) is often erratic and superficial. While some bridges and permanent links have been recently established (e.g., through multimedia programs), huge conceptual and behavioral gaps and mismatches are clearly observable.

The author of this paper, who is completing his 30-year career as a professor of engineering (engineering physics), can testify that "hard" and "soft" sides of the campus are often unaware of even major events happening at the other end. Likewise, faculty and students from both "camps" do not generally display much interest in each other. Even within the same university the top and established professors from one side often do not know the names of top scholars from the "other" side. In the matters of funding and priority settings there is often a sentiment that the "other side" gets too much while "we" are disfavored. 


\subsection{Interdisciplinary connections}

While the exceptions from the above scenario undoubtedly do exist, the perception of the hard-soft split remains broadly accepted as was earlier emphasized by Charles Percy Snow (1905-1980) in his famous Rede Lecture on "Two Cultures" (1959) [1, 2]. This split between "soft" and "hard" knowledge is not limited to the realm of academia, but permeates other social strata.

\subsubsection{Third culture}

Fortunately, in recent years this split is somewhat mitigated by the emergence of the "Third culture" [2] and its connection to a general social milieu. The latter term is used to designate a variety of intellectual and pseudo-intellectual ideas and practices which, in one way or the other, touches a significant proportion of the population.

Thus, apart from individual ideas and preferences, a majority of engineering design professionals, through education, experience and their day-to-day work are often connected to intellectual and cultural diversity through numerous and ever-changing dynamic links. While the degree and focuses in such connection greatly differ from case to case, their influence can be traced in several ways, as discussed further in this paper.

\subsubsection{Facts and inferences}

In tracing interdisciplinary connections facts and inferences are, as a rule, not clearly demarcated. Often, the influence of a particular trend or type of art on the products of ED can be suggested only as plausible guesswork.

\section{Definitions and qualifications}

We live in the world of ever-changing trends, fashions and buzzwords. They change at a compressed time-scale, often just a few years. Nowadays, one of the most popular household ("cool") terms is the word quantum. Its use goes well beyond its original domain of quantum physics as such. Now we hear and read about "quantum health", "quantum investments", "quantum leaps", and even consumer brands like "quantum vodka".

In this paper we focus on such general extensions of quantum ideas as quantum nonlocality, quantum entanglement, and quantum information; the latter related to quantum computing and information as a physical concept.

\subsection{Visionary art and virtual reality}

This paper discusses links between ED and such diverse, and yet interlinked, notions as quantum nonlocality, nature of information, nanotechnology and arts. Visionary art - that is an art with philosophical and inquisitive substrate, such as paradoxical and surrealistic art - is especially illustrative in this regard.

Recently emerged interactive and dynamic art, proliferating through multimedia and Internet, may be seen as a superposition (a mixture) of visionary art and virtual reality in a more traditional sense. 


\subsubsection{Quantum information as encompassing idea}

All inventions and artistic products come from our consciousness, our imagination and the repositions of ideas and pattern which our mind can possess and process. Metaphorically, one can refer here to some abstract depository of patterns and potential designs somewhat akin to imaginary "Library of Babel" (collection of all possible books) [3, 4].

\subsection{Over-view versus comprehensive review}

This paper, due to its size and focusing, does not pretend to be anything like a comprehensive compendium on artistic and conceptual links to ED. It is rather a sketch of a few noticeable points without an attempt to provide an exhaustive referencing to published sources.

\subsubsection{Printed literature versus web sources}

In fact, due to the ever-growing shift from printed literature to WWW sources, the role of formal referencing is declining. Many thoughts and ideas are often quoted without proper referencing to particular authors and originators. Likewise, the issues of the copyright and intellectual property become more and more complicated and convoluted. The very concept of authorship often becomes fuzzy and poorly identified. This may appear unfair to many deserving authors, yet the whole tide is so massive that it can hardly be contained. We just have to take it as a part of modern life. Some critics indicate that the very idea of digital copyright is inconsistent and fallacious.

Thus, both in the realm of printed literature (books, journals, published conference proceedings, etc) as well as WWW sources (which are often unattributed or misattributed), a comprehensive search is all but unrealistic.

\section{It from Bit paradigm}

The present author believes that the notion of It from Bit was introduced by the physicist John Archibald Wheeler (1911-2008), one of the most original and insightful thinkers of the 20th century, who only by some curious omission has "missed" his well-deserved Nobel Prize.

One of the key points of Wheeler's work was his emphasizing of information as a physical category. Everything, in this view, comes from the pure information, pure binary strings, or YES and NO answers. In this vein, arts, ideas and ED are not exceptions. The ideas akin to the It from Bit paradigm are discussed by many authors (see some references in [3]) and recently the very term was paraphrased by Seth Lloyd into a quantum domain ([5], p. 175) in a form of It from qubit (qubit = quantum bit).

\subsection{It from Bit in the context of art}

While people of art are usually reluctant to discuss their techniques and products in terms of physics and information, once in a while they display implicit recognition that their creativity is grounded at some higher level of existence. 
Subjective assessment of that may range from utter mysticism and spiritual enchantment (e.g., El Greco, Van Gogh, some Romantic paintings) to fathomless depths of human psyche (surrealism). As a more down-to-earth encompassing cliché we can recall a known quote assigned to Michelangelo. When asked how he creates his statues, his reply was that he does not actually "create" any of them, all he does is removing extra marble which "traps" them inside the block. This and other similar accounts point to the Ideal Platonic World of forms and numbers as an eternal and infinite depository of patterns and structures from which all creations arise either by natural evolution or human ingenuity. Time changes the terminology (e.g., "quantum field" replaces "Platonic World") but has little effect on the essence of message.

\subsubsection{Implication of It from Bit to engineering design}

Typically, ED as well as architectural projects starts from conceptualization. The key elements are goals and available resources. The latter includes "know-how" of the day. Yet, such visionary inventors as "grandfather of digital computer" Charles Babbage (1791-1871), or electricity maverick Nicola Tesla (1856-1943), or Buckminster Fuller (1895-1983), who has designed a Geodesic Dome - a structure which later has found its analogy in a form of carbon C60 molecule ("buckminsterfullerene" or "buckyball"), often jumped over of what was known and available at their days and made technological breakthroughs which were well ahead of their time.

\subsubsection{Archetypes and meme principles}

In discussing the typologies of ED the notion of "archetypes" and "meme" can serve as useful guidelines. The first notion (archetype) is usually associated with Carl Gustav Jung (1875-1961) who productively used it in the context of psychology and spirituality. Yet, in recent years, the term percolated into some other ("hard") areas, especially due to the science of chaos, non-linear dynamics and complexity $[6,7]$.

Notion of "meme" was introduced by Richard Dawkins (b. 1941) in 1976 in genetics [8], but, similarly to 'archetypes', has now spread well beyond it original territory and is used to analyze fads, fashions, trends, catch words and other social trends. Proliferation of it is greatly enhanced by the Internet.

4.1.2.1 Archetypes in engineering design can be defined in several ways. One is to relate them to a particular major area of ED, like laser engineering, fiberoptics, micromachinery (MEMS), bio-nano-technology, etc. Each of these (sub) areas of ED have their own home-culture, quality and value scales, and types of connections to a user sector. The paradigm of virtual reality and its more specific manifestation in a form of CAD (computer aided design) opens another archetypical vista. This can be referred to products of ED as to the designers themselves. For example, it is almost a universal observation that practical "dirty hands" inventors (i.e., actual "doers", like T.A. Edison or A.G. Bell) are usually differ psychologically (and often, personally) from "paper only" inventors (or, nowadays, people doing CAD only). 
4.1.2.2 Meme in engineering design can likewise be defined as designating some current trends, especially in the area of esthetic appeal of the design products (e.g., consumer electronics, photo cameras, computer accessories, shapes of trendy cars, all kind of packaging, etc). To what extent ED "memes" can be patented or trademarked remain generally a gray area of law and legal practice because, as a popular cliché has it, "ideas cannot be patented, but their application to specific products can".

4.1.2.3 Synchronicity and "esthetic resonance" between artistic vision and objectives of engineering design is another largely unexplored area. Artistic images, especially popular and "cool" ones, are in abundance all around us and people (say, engineering inventors and designers) are constantly bombarded by them. This applies even to those people who deny any special interest in arts. And yet, Zeitgeist of the dominant esthetic culture inevitably affects all creative people, though to a vastly different degree.

\section{Artists as design inspirators}

Here we mention a few selected figures in the world of arts, who often or occasionally, are seen as noticeably affecting engineering or architectural design.

\subsection{Design envisions in pre-modern art}

When a new technological development appears on the scene, one can seek for possible sources of inspiration available at that time which affected the invention. Almost never is there direct evidence of such an interaction and most of these inferences are bound to remain at the level of plausible conjectures. One example is eye glasses. While the exact inventor(s) of them is/are unknown, most point to 13 th century Italy (Pisa or Venice) where the artistic glass-blowing was an established industry. Was the invention of eye glasses an act of "design engineering" (first idea, then the implementation), or it was a spontaneous case of "serendipity", remains a guesswork, but soon eye glasses appear on paintings of several artists (e.g., Tommasa da Modena, Jan Van Eyck, Carlo Crivelli).

\subsubsection{Middle ages and renaissance}

Apart from already mentioned titans Leonardo and Durer, interesting insight are found in the art of Hieronymus Bosch (ca. 1450-1516) with his images of fantastic flying machines with wings - perhaps, some distant envisioning of modern aircrafts. Some inspiration can be found in the exotic art of Giuseppe Archimboldo (1527-1593) whose images of human faces are composed from a peculiar variety of objects - perhaps, some precognition of chaos and complexity ideas of today.

Seventeen century witness a spectacular surge of artistic activity in the Republican Netherlands where many paintings contained boats and ships. Due to the ubiquity of Dutch painting at this period (almost every Dutch home had pictures) the connection between shipbuilding and marine painting comes as a natural assumption. 


\subsubsection{From classicism to impressionism}

The 18th and 19th centuries witnessed several trends in arts, Classicism, Romanticism and Impressionism being the major ones. While the most visible effects of them can be traced in architectural and applied design, some links to science and engineering can also be indicated.

5.1.2.1 Eighteenth century European art was dominated by late Baroque and Classicism while science and technology of this period experienced a quantum leap. Breakthrough developments in chemistry, observational astronomy, first experimentations with electricity, hot air balloons, and, most of all, the invention of the steam engine - all have some accounts in visual arts and the interactive links can be traced in some cases. For example, the invention of bifocal glasses by Benjamin Franklin (in 1784), or a widespread use of camera obscura (precursor of photography, although its appearance can be traced to earlier times) can be put in a co-junction with artistic experiments with ambiguous images.

5.1.2.2 Romanticism of the first part of 19th century (Caspar David Friedrich, Thomas Cole, etc) had effects on the architecture, furniture design, cloth fashions, etc. Pictures such as Architect's Dream (T. Cole, 1840, Toledo Art Museum) and artistic competitions prior to International Exhibitions have eventually led to such marvels as Eiffel Tower (1889).

Thomas Cole's The Titan's Goble (1833, Metropolitan Museum, New York) encapsulates the idea of build-in worlds, or separate universes, each with its own existence. In modern times this vision resonates with cosmological ideas of multiple universes which are thought to be chain-generated at ultra-small (Planck) scales. On a technological side it is akin to quasi-independent subsystems in complexity theory and operation of various blocks in electronic devices or sub-routines in computer programs.

5.1.2.3 Impressionism since it emergence in 1860-s and quickly growing popularity, is a testimony of fragmentation of knowledge and culture and inability of a single individual to hold it all. Hence, a need for a bird-view, bottom-lining and a frequent urge to make quick decision based more on impressions than on weighted analysis of the facts. On ED side it is illustrated by the fact that the huge proportion of patents issued in the 20th century turned out to be dead-ended and unused.

5.1.2.4 Pointillism, one of the outfits of impressionism (George Seurat, 18591891, Paul Signac, 1863-1935) has recently found an interesting application to the detection of faults in computer systems, specifically, for the detection of network anomalies caused by overload or corrupted systems [9]. Pointillistic approach permeates design of pixel-based electronics from TV, computer screens and digital cameras to high-way and shopping malls tabloids.

\subsection{Modern period (20th century)}

Interaction between the worlds of arts and technology in the 20th century was further fostered by two major factors. One is the explosive development of mass 
communications and means of information technology which led to the emergence of multimedia as an interdisciplinary umbrella concept. The second factor is a vast intensification of contacts between people across all traditional barriers - national, ethnic, cultural, professional, economical and educational.

\subsubsection{From art nouveau to abstractionism}

Most explicit examples of art-design interaction in this period are found on the side of visionary arts, especially, surrealism. Several styles, which have some connection to surrealism, but are not surrealism in exact sense, also ought to be mentioned.

5.2.1.1 Art Nouveau and Art Deco are largely esthetic styles which, apart from effects on architecture (especially, public and commercial buildings), had noticeable influence on the development of packaging, shapes and decoration of mass consumer products from telephones and automobiles to recreational facilities and high-rise elevators.

5.2.1.2 Constructivism and Cubism (Braque, early Picasso, etc) by their very "technological" and "futuristic" allure, had some real, as well as pretentious, effects on the development of modern vision of design of complex industrial systems, like assembly lines and some architectural solutions. Similar, and even sometime more vigorous and futuristic, development has taken place in Russian/Early Soviet vanguard art of 1910-1930s.

5.2.1.3 Modern abstractionism as represented by such names as Malevich, Kandinsky, Pollack, Mondrian, etc., is another trend affecting engineering thinking and several subtle ways. Testimony to that is the fact that samples of this art often decorate offices of high-tech designers and executives. Lay-outs of microelectronic circuits on silicon chips may well be an example of analogies taken from abstract art.

In terms of conceptual and practical impact, such creators as Yves Tanguy (1900-1955) or Joan Miro (1893-1983), whose art stylistically belongs to both abstractionism and surrealism, illustrates the principle of diversified units, which philosophically is akin to Leibnitz monads. It lies at the basis of the design of complex interactive informational systems from fiber-optic communication networks to structure of hyperlinks in WEB search engines.

\subsubsection{Surrealism and paradoxical art}

Surrealism and paradoxical art (Giorgio De Chirico, Max Ernst, Salvador Dali, Rene Magritte, Maurits Escher, Oskar Reutersvard, etc) is another powerful source of creative inspiration [10]. Of those, Dali and Escher are probably most stimulating in terms of creativity and design ideas.

5.2.2.1 Salvador Dali (1904-1989) is one of the most provocative artists of the 20th century. His artistic experiments on multidimensional realms, transitory character of his objects and his twisting of a space-time realm, like images of "liquid time" (The Persistence of Memory, 1931), or his fascination with atomic physics (e.g., Melancholic Atom and Uranium Idyll, 1945 or Leda Atomica, 
1949) resonate with novel ideas of fundamental and applied physics, such as relativity, quantum parallelism and backward causation along "looped" world lines (J.A. Wheeler). Recent developments in quantum computing are well in tune with these images and ideas. One of his last pictures (The Swallow's Tail Series on Catastrophes, 1983) represents a mathematical image taken from the Theory of Catastrophes which is used in design engineering and structural stability assessment.

5.2.2.2 M. C. (Maurits Cornelis) Escher (1898-1972) is a Dutch artist whose lithographs are notoriously popular in science and engineering community [11, 12]. His famous Belvedere (1958) is an example of a construct which is topologically impossible in a 3-dimensional space, yet appear fully realistic. Quantum computing systems rest on a similar notion of "realizable impossibility", where the co-existence of mutually exclusive alternative states becomes allowed by the superposition principle. In Cubic Space Division (1952) Escher foresees 3-dimensional integration in nanoelectronics which came to the stage of design and implementation in later decades.

5.2.2.3 Ambiguous images, such as a well-known picture which visually alternates between young and old woman (Edwin Boring, 1941), have interesting conceptual resonance with design of bistable electronics like tunnel diodes and fast switching elements in which a small bias (weak signal) can trigger a device into one of two quasi-stable states.

\section{Physical concepts in engineering design}

This section gives an overview of selected issues at the intercrossing of fundamental physical concepts and dynamics of ED.

\subsection{Are laws of physics discovered or invented?}

The ideas of mathematics and set theory appear somewhat easier to discuss in terms of their origin and foundations than the laws of physics. Contrary to physics, mathematics is not based on any "hardware" (such as matter, particles, energy and other typical categories of physics). This is often molded to a contention that in some "other universe" the laws of physics and forms of matter (e.g., types of elementary particles and their parameters) may be very different from ours, but, yet, the mathematics will be the same. Such facts of mathematics as, for example, list of prime numbers, is eternal, universal and unchangeable. In this regard, ED, which in certain aspects draws from eternal ("Platonic") forms and patterns, stands half-way between mathematics and physics.

\subsubsection{Physics relies on experimentation}

To what extent physics is "discovered" or "invented"? In physics, with its prime reliance on experimentation, this issue still remains open for discussions and contemplations. In the tradition of ancient and medieval disputes between realism and nominalism, modern reflection of "discovered or invented" issue in 
physics transpires into such areas as particle physics, inflationary cosmology, multiple universes and fundamental mathematics and logic such as set theory, continuum hypothesis or Godel's undecidability theorems.

Using all possible permutations of elementary particles Max Tegmark calculates that the next "Hubble Bubble" (observable universe) identical to ours is some $10^{\wedge} 10^{\wedge} 150 \mathrm{~km}$ away (tower exponent notation) [13]. However, the true versus approximate identity of both Hubble Bubbles (and hence, reality of Eternal Return scenarios of Mircea Eliade, etc), rests on such issues as discrete versus continuous spectrum of states of Hubble Bubble and/or exact versus approximate identity of like elementary particles [14].

6.1.1.1 Universe as a quantum computer - a model in which digitization spreads over one or more Hubble Bubbles $[5,14]$ - or "simulation argument" [15], application of which to ED is still awaiting attention, pictures universe as a functioning quantum computer, which allows parallel processing at different regions of the megaverse [16-18].

Other pertinent model, which apparently circumvents Eternal Return trap, is Born-von Karman quasi-periodical chaining of (infinite) network of "elementary" Hubble Bubbles. This lifts minimum and maximum energy cutoffs and opens the way to genuine infinite innovations in the (mega)verse. This, as Freeman Dyson posits it, makes the world truly Infinite in All Directions [19] with a room for infinite creativity and unrepeatability along infinite (uncountable) set of "world lines".

\subsubsection{Quantum stimulation of human creativity}

Quantum physics points to some possibilities in terms of human interconnectedness which may account for some bursts of creativity. The latter, in turn, can be stimulated by subtle and random inputs. This quantum interconnectedness is illustrated by the so called Wigner friend paradox related to the role of conscious observer in quantum measurements. As Grib \& Rodrigues put it, "the idea of world consciousness says that when I am conscious of something external, I am just participating in some nonlocal world consciousness, where introspection takes place" ([20], p. 53).

Is-spite that such an idea may, at first glance, seem too far-fetched, nonlocal nature of quantum world gives a solid basis for the validity of such hypotheses. Recent developments in quantum computing, quantum information (teleportation) and wave function collapse theories [3, 5, 16-18] point in this direction. As a manifestation of these effects, the stimulation of engineering creativity and new design ideas can come from subtle esthetic stimulations by various artistic forms - a connection which often cannot be properly described in exact facts and, yet, has numerous personal accounts and anecdotal reports.

\section{Conclusion}

The interaction and creative enrichment between arts, engineering activities (design, architecture, civil and environmental engineering, etc) and fundamental 
physical concepts seems to be almost self-evident across the ages and cultures [21-23]. However, due to subtle nature of these interactions they rarely, if ever, can be convincingly documented.

This paper took a somewhat more pro-active approach to the problem of the said interactions and ventured to offer a few links which may not be properly discussed or even noticed as yet. The issue is additionally complicated by the social constrains coming from the copyright and patent systems which often discourage people from making explicit attributions to their sources of inspiration and creativity. In this view the proliferation of combined educational college programs like Art and Engineering, Multimedia, Architectural Design, etc., and a broad choice of liberal art courses, can foster the strengthening of arttechnology links.

\section{References}

[1] Snow, C.P., The Two Cultures, Cambridge University Press, 1993.

[2] Brockman, J., The Third Culture: Beyond the Scientific Revolution, Simon \& Schuster, N.Y., 1995.

[3] Berezin, A.A., "Natural evolution, human creativity and engineering design in the context of paradigms of modern physics", Design and Nature IV, ed. C.A. Brebbia, WIT Press: Southampton, Boston, pp. 301-310, 2008.

[4] Bloch, W.G., The Unimaginable Mathematics of Borges' Library of Babel, Oxford University Press, 2008.

[5] Lloyd, S., Programming the Universe: Is the Universe actually a Giant Quantum Computer?, Vintage Books (Random House), New York, 2007.

[6] Berezin, A.A. \& Gridin, V.V., "Some aspects of air pollution in historical, philosophical and evolutionary context", Air Pollution XVII, eds. C.A. Brebbia \& V. Popov, WIT Press, Southampton, Boston, pp. 341-352, 2009.

[7] Mitchell, M., Complexity: A Guided Tour, Oxford University Press, 2009.

[8] Dawkins, R., The Selfish Gene, Oxford University Press, 1976.

[9] Xie, Y., Kim, H., O’Hallaron, D., Reiter, M. \& Zhang, H. "Seurat: A Pointillist Approach to Anomaly Detection", Proceedings of the Seventh International Symposium on Recent Advances in Intrusion Detection (RAID 2004), Sophia Antipolis, French Riviera, September 2004.

[10] Seckel, A., Masters of Deception: Escher, Dali \& Artists of Optical Illusion, Sterling Publishing Co., Inc., New York, 2004.

[11] Locher, J.L. (ed), The World of M.C. Escher, Abradale Press (Harry N. Abrams, Inc.), New York, 1988.

[12] Coxeter, H.S.M., Emmer, M., Penrose, R. \& Teuber, M.L. (eds), M.C. Escher: Art and Science, Elsevier Science Publishers B.V., Amsterdam, 1986.

[13] Tegmark, M., "Parallel Universes", Scientific American, Vol. 288 (5), pp. 41-51, 2003.

[14] Berezin, A.A., "Identity of Particles and Continuum Hypothesis", Bulletin of the American Physical Society, Vol. 46 (2), p. 145, 2001. 
[15] Bostrom, N., “Are you living in a computer simulation?", Philosophical Quarterly, 53 (211), pp. 243-255, 2003.

[16] Deutsch, D., Fabric of Reality, Allen Lane (Penguin), London, 1997.

[17] Penrose, R., The Road to Reality: A Complete Guide to the Laws of the Universe, Vintage Books (Random House), London, 2005.

[18] Carr, B. (ed), Universe or Multiverse?, Cambridge University Press, 2007.

[19] Dyson, F.J., Infinite in All Directions, Harper \& Row, New York, 1988.

[20] Grib, A.A. \& Rodrigues, W.A., Nonlocality in quantum physics, Kluwer Academic/Plenum Publishers, New York, 1999.

[21] Berezin, A.A. \& Gridin, V.V., "Complexity theory in management of heritage architecture preservation", STREMAH 2009 (Structural Studies and Maintenance of Heritage Architecture XI, ed. C.A. Brebbia, WIT Press, Southampton, Boston, pp. 255-266, 2009.

[22] De Camp, L.S., The Ancient Engineers, Ballantine Books, New York, 1988.

[23] Schillinger, J., The Mathematical Basis of the Arts, Philosophical Library, New York, 1948. 